\title{
Formación de Precios en un Mercado Artificial de Doble Subasta Continua
}

\begin{abstract}
Resumen
En este trabajo se estudia la formación de precios en un mercado artificial de doble subasta continua con agentes heterogéneos, tanto en términos de eficiencia informativa como en términos de sus propiedades estadísticas. A diferencia de otros mercados artificiales propuestos en la literatura, en este mercado existe información asimétrica tanto ex-ante como ex-post, puesto que los agentes no informados observan únicamente precios de transacción pasados. En consecuencia, su capacidad predictiva sobre el proceso fundamental está limitada por el grado de eficiencia informativa de los precios de transacción, endógena al mercado. Nuestro mercado es capaz de replicar los hechos estilizados observados comúnmente en las series reales de rendimientos (colas gruesas, persistencia en la volatilidad, correlación serial y efectos ARCH), y nos permite extraer predicciones teóricas con respecto al efecto de la distribución de la población de agentes sobre dichas propiedades estadísticas. Una diferencia del trabajo es la modelización del precio fundamental como un proceso estocástico, lo cual permite calibrar los parámetros del mercado artificial a datos reales. La principal conclusión del trabajo es que el mecanismo de doble subasta continua permite un elevado grado de eficiencia informativa cuando existe información asimétrica, además se observa que la eficiencia del mercado puede mejorar introduciendo inversores sin información privilegiada pero que explotan la información contenida en los precios de transacción pasados (por ejemplo, analistas técnicos y agentes con capacidad de aprendizaje), aunque ello disminuya el valor de la información privada y por tanto la riqueza de aquellos con información privilegiada.
\end{abstract}

Palabras Clave: Microestructura de Mercados, Mercados Artificiales, Eficiencia, Aprendizaje

JEL: G12, G14, D83. 


\section{Introducción}

La microestructura de mercado analiza, entre otros aspectos, el impacto que la estructura de mercado o conjunto de reglas que gobiernan el funcionamiento de un mercado tiene sobre el comportamiento de los inversores y los costes que estos inversores sufren a la hora de realizar transacciones.

La investigación en microestructura se ha ocupado principalmente de cuatro grandes cuestiones (Madhavan, 2000):

- Cómo se produce la formación y el descubrimiento de precios.

- Cómo afectan las reglas del mercado a la formación de precios.

- Qué papel juega la transparencia del mercado.

- La relación de la microestructura con las otras áreas de investigación en Finanzas, como la valoración de activos.

Los estudios teóricos han permitido responder en gran medida a las cuestiones anteriores. Sin embargo, el análisis teórico no puede captar en su plenitud la complejidad de los mercados reales poblados por un alto número de agentes heterogéneos, que interactúan entre ellos repetidamente, y que aprenden y evolucionan con el paso del tiempo. La génesis de estas heterogeneidades puede ser muy diversa: los agentes pueden diferir en cuanto a su grado de racionalidad, aversión al riesgo, preferencias, dotación económica o informativa. En tales condiciones, los modelos se vuelven intratables y ni siquiera la existencia de equilibrio puede garantizarse.

Por otra parte, los trabajos empíricos explotan el vasto caudal de información generado diariamente en los mercados reales. El alcance de sus resultados, sin embargo, está limitado por la imposibilidad por parte del investigador de controlar las condiciones en que dichos resultados se han obtenido. Por ejemplo, en los estudios empíricos no es posible estudiar la eficiencia informativa de los precios de distintos mecanismos de negociación, dado que el verdadero valor del activo no es observable en dos tipos de mercado distintos al mismo tiempo (Theissen, 2000).

La Economía Experimental (véase, por ejemplo, Ackert et al., 2001) ha contribuido a resolver estas deficiencias, permitiendo, por una parte, mayor realismo que los modelos teóricos, puesto que en los experimentos se emplean agentes humanos y, por otra parte, un alto grado de control sobre las condiciones del experimento inexistente en los estudios empíricos. A pesar de ello, los mercados experimentales sufren una limitación importante: ni la motivación, ni las actitudes frente al riesgo, ni 
las expectativas, ni el comportamiento de aprendizaje general de los agentes son controlables (Chan et al., 2001; Yang, 2002).

En este contexto, los mercados financieros de agentes computacionales o mercados financieros artificiales, basados en simulación, emergen como una novedosa y prometedora herramienta para el estudio de la Microestructura. ${ }^{3}$ Concretamente, distintos trabajos han analizado entre otros aspectos el efecto de la estructura de mercado sobre la eficiencia informativa de los precios (Gode y Sunder, 1993), la dinámica de los precios y su eficiencia (Chiarella y Iori, 2002), o, más recientemente, el efecto de la sincronización de las acciones de los agentes (Iori, 2002). A pesar de su flexibilidad, los mercados financieros basados en agentes computacionales deben entenderse como una metodología complementaria a las señaladas anteriormente y mas concretamente a los estudios teóricos. Entre las limitaciones de este tipo de aproximación metodologica debemos destacar dos. Por un lado, los mercados computacionales no permiten la modelización de comportamientos de tipo estratégico por parte de los agentes, lo cual obliga a suponer racionalidad limitada. Por otro lado, la indudable flexibilidad hace que en algunas ocasiones sea mas difícil extraer conclusiones frente a los modelos teóricos de menor complejidad.

En este trabajo, presentamos un nuevo modelo de mercado artificial basado en agentes computacionales que puede utilizarse como marco realista para el estudio cualitativo y cuantitativo de problemas de microestructura. En concreto, proponemos un mercado en el que el valor fundamental del activo varía entre períodos de acuerdo a un proceso estocástico que sólo es observado (con ruido) por un subconjunto de agentes, mientras que el resto de agentes no observa el valor fundamental, ni siquiera ex-post, y forman sus expectativas a partir de precios de transacción pasados utilizando o bien el análisis técnico o bien redes neuronales artificiales.

Encontramos, en primer lugar, que el mercado de doble subasta continua permite una diseminación razonable de la información privada en posesión de algunos agentes. Es precisamente la eficiencia informativa de precios pasados la que permite que el análisis técnico y especialmente el aprendizaje mediante redes neuronales artificiales produzcan estimaciones de precios mejores que el simple paseo aleatorio, lo cual a su vez, mejora la eficiencia global del mercado, pero disminuye las rentas de los agentes con información privada.

\footnotetext{
${ }^{3}$ Véase LeBaron (2000) para una revisión de la literatura.
} 
Por otro lado, nuestro mercado reproduce los hechos estilizados observados en los mercados reales (leptocursis, no normalidad, predecibilidad, efectos ARCH) tanto cualitativa como cuantitativamente. Podemos, por tanto, estudiar el efecto de la composición de la población de agentes o de la distribución de información sobre las propiedades estadísticas de los rendimientos o el volumen de transacciones en el mercado.

El resto del trabajo se organiza de la siguiente manera: En la Sección 2 se revisa la literatura y se explica la contribución del trabajo; En la Sección 3 se describe la construcción de un mercado artificial de agentes computacionales heterogéneos; En la Sección 4 se muestran los resultados de las distintas simulaciones llevadas a cabo; Y, por último, en la Sección 5 se presentan las principales conclusiones del estudio y se discuten posibles extensiones futuras.

\section{Revisión de la literatura y contribución}

Uno de los mercados basados en agentes computacionales más citados que incluye la posibilidad de aprendizaje es el Mercado de Valores Artificial del Instituto de Santa Fe (Arthur et al., 1997; LeBaron et al., 1999; LeBaron, 2001; LeBaron, 2002). Este mercado está basado en los modelos neoclásicos de agentes racionales como Bray (1982) o Grossman y Stiglitz (1980). En él, los inversores forman expectativas sobre el precio del activo en el siguiente periodo y derivan sus demandas óptimas del activo en función de dichas expectativas. La innovación del mercado de Santa Fe consiste en la introducción de heterogeneidad y aprendizaje en la formación individual de expectativas. Los autores encuentran que el mercado de Santa Fe es capaz de reproducir los hechos estilizados observados en las series financieras reales: la distribución de rendimientos es leptocúrtica, la volatilidad persistente, y los rendimientos muestran cierto grado de predecibilidad.

El mercado que proponemos en este trabajo está inspirado en el de Santa Fe así como en las contribuciones recientes de Yang (2002) y Chan et al. (2001). Yang (2002) considera un mercado de agentes computacionales similar al de Santa Fe, pero con la particularidad de que los agentes con capacidad de aprendizaje forman sus expectativas de precios a partir de precios de transacción anteriores y dividendos pasados empleando redes neuronales artificiales. Por su parte, Chan et al. (2001) presentan una variación del 
mercado de Santa Fe, introduciendo un sistema de doble subasta continua frente a la subasta walrasiana implícita en el mercado de Santa Fe.

Una de las principales desventajas de los trabajos mencionados radica en la dificultad de calibrar los mercados a datos reales, ya que el supuesto de valor fundamental constante o las distribuciones propuestas para el mismo, no son verosímiles. En palabras de LeBaron (2002), el modelo no está alineado con series financieras reales, lo cual no impide la extracción de conclusiones cualitativas, pero sí hace imposible valorar el éxito o fracaso del modelo en términos cuantitativos. Nosotros proponemos modelizar directamente el proceso para el verdadero valor del activo, es decir, su precio fundamental, en lugar de suponer que es constante o función del proceso generador de dividendos. Ello permite dotar de mayor realismo al modelo y generar series de precios de transacción que se asemejen a las reales no sólo cualitativa sino también cuantitativamente. En concreto, en nuestro mercado la dinámica del valor fundamental del activo está gobernada por un proceso de Ito. La ventaja de emplear un proceso en tiempo continuo es que una vez especificados los parámetros del mismo (términos de deriva y de difusión) es posible generar precios sobre intervalos de tiempo arbitrariamente pequeños. A pesar de que en principio cualquier difusión es valida, en este trabajo escogemos un proceso browniano geométrico debido a que la distribución de rendimientos bajo dicho proceso no presenta correlación serial, leptocurtosis o heteroscedaticidad condicionada, luego, si dichas propiedades estuvieran presentes en la serie de precios de transacción, ello sería debido exclusivamente a la interacción de los agentes y al sistema de negociación escogido.

Por otro lado, a diferencia de los mercados de agentes computacionales propuestos en la literatura, que permiten a los agentes actualizar sus expectativas usando información sobre precios y sobre la realización del estado de la naturaleza, en nuestro mercado existe asimetría informativa sobre el valor fundamental del activo no sólo exante sino también ex-post, de forma que la única forma de aprendizaje posible para los agentes no informados, es a través de precios de transacción pasados. Pensamos, por un lado, que debe explorarse en este contexto la posibilidad de extraer información de precios pasados y el efecto que tiene sobre la eficiencia informativa del mercado, que a su vez determinará la capacidad de aprendizaje futura de los agentes. Por otra parte, este enfoque nos parece más consistente con los mercados reales, en los cuales los inversores no llegan a conocer el verdadero valor del activo o precio fundamental. 
Por último, comparamos por primera vez el efecto de la presencia de agentes técnicos y agentes que emplean redes neuronales artificiales, tanto cuando estos agentes compiten por separado contra agentes informados y no informados, como cuando compiten entre sí.

\section{Un mercado financiero de agentes computacionales}

A continuación se presenta el mecanismo de negociación así como la composición del mercado en cuanto a los agentes que participan en el mismo.

\subsection{Estructura del mercado}

Nuestro modelo prescinde del papel del especialista o market maker centralizado ya que las órdenes se casan automáticamente. La forma de replicar un mercado real de doble subasta continua sigue la propuesta por Chan et al. (2001). Cada período de negociación (un día), está compuesto de 5 rondas, lo que implica que cada agente acude 5 veces al mercado en un período. El orden que ocupa cada agente en cada ronda es aleatorio y sigue una distribución uniforme. Llegado su turno, cualquier agente puede introducir una orden límite, es decir, enviar un precio de oferta o de demanda al sistema. El precio enviado prevalece en el sistema sólo si mejora el precio anterior, es decir, si reduce la horquilla entre el precio de demanda y de oferta. Alternativamente, el agente puede introducir una orden de mercado, es decir, comprar al precio de oferta que permanezca en el sistema, o vender al precio de demanda. En cualquier caso, y siguiendo a Chan et al. (2001), la orden siempre es por una unidad del activo. Asimismo, no se permite ventas al descubierto ni pedir prestado, y los agentes deben satisfacer en todo momento su restricción presupuestaria. Los agentes almacenan efectivo en un activo libre de riesgo que paga una rentabilidad neta nula.

En la subseccion 3.3 se describe en detalle el procedimiento de generecion de órdenes.

\subsection{Precio fundamental del activo}

La dinámica del verdadero valor o precio fundamental del activo con riesgo está gobernada por un proceso browniano geométrico:

$$
d P_{f, t}=\mu P_{f, t} d t+\sigma P_{f, t} d W_{t}
$$


donde, $P_{f, t}$ representa el precio fundamental del activo en el periodo $t, \mu$ es el coeficiente de deriva del proceso, o rentabilidad instantánea esperada del activo, $\sigma$ es el coeficiente de difusión o volatilidad local, y $\mathrm{W}_{\mathrm{t}}$ es un movimiento browniano estándar. Bajo dicho proceso, la distribución condicionada de precios futuros al precio del periodo t-ésimo es lognormal:

$$
\ln P_{f, t+\Delta} \mid P_{f, t} \sim \phi\left(\ln P_{f, t}+\left(\mu-\frac{\sigma^{2}}{2}\right) \Delta, \sigma \sqrt{\Delta}\right)
$$

donde $\Delta$ es el intervalo temporal entre dos períodos en términos anuales (que fijaremos en $250^{-1}$ ) y $\phi$ denota la función de densidad normal. Por tanto, el rendimiento continuo del precio fundamental entre dos períodos, calculado como la diferencia de logaritmos de precios, se distribuye de acuerdo a una normal serialmente independiente y con varianza constante.

\subsection{Agentes computacionales}

Todos los agentes en el mercado son "miopes” y neutrales al riesgo. ${ }^{4}$ Su función objetivo, por tanto, es el valor esperado de su riqueza al final de cada período de negociación.

El agente i-ésimo, por tanto, obtiene una predicción del precio de la acción al final del periodo de negociación en que se encuentra, $P_{t}^{i}$, y compra (vende) siempre que el precio de oferta (demanda) del sistema sea inferior (superior) a su predicción. También puede enviar un precio de oferta (orden límite de venta) uniformemente distribuido entre $\left(P_{t}^{i}, P_{t}^{i}+S\right)$, donde $\mathrm{S}$ se fija en 0,5 . El parámetro $\mathrm{S}$ juega un doble papel: por una parte un mayor valor de S aumenta el beneficio de la operación y por otra parte limita la probabilidad de ejecución de la orden límite. Por último el agente puede enviar un precio de demanda (orden límite de compra) uniformemente distribuido entre $\left(P_{t}^{i}-S, P_{t}^{i}\right)$. El Cuadro 1 resume el procedimiento de generación de órdenes.

Los agentes del mercado se pueden dividir en dos clases: informados y desinformados. Mientras que los informados observan el valor fundamental con ruido, los

\footnotetext{
${ }^{4}$ Mientras que el supuesto de miopía es común en esta literatura, la neutralidad al riesgo nos garantiza que la valoración del activo con riesgo por parte de cada agente es igual a la predicción que dicho agente hace de su precio. Si, por el contrario, el agente fuese averso al riesgo, su valoración sería inferior debido a la prima de riesgo. Ello nos permite centrarnos en "diferencias de opinión” y abstraernos del problema de las distintas actitudes frente al riesgo.
} 
desinformados tratan de predecir el precio a partir de la observación de los precios de transacción pasados. Los agentes desinformados se diferencian entre sí en la forma en que obtienen la predicción del precio fundamental del activo. Mas concretamente, en nuestro mercado consideramos la existencia de:

1. Agentes Informados o Fundamentales (AF). Observan el precio fundamental del activo, más un ruido aleatorio distribuido uniformemente entre el -1\% y el 1\% del precio fundamental, $P_{f, t}$, y piensan que el precio de transacción debería converger al fundamental:

$$
P_{t}^{i} \sim U\left(0.99 P_{f, t}, 1.01 P_{f, t}\right)
$$

Estos agentes pueden ser considerados como “insiders” y por tanto, nos permite analizar diferentes aspectos en un mercado caracterizado por agentes con información privilegiada.

2. Agentes con inteligencia cero (AIC). Su pronóstico del precio fundamental de la acción es una realización de una variable aleatoria distribuida uniformemente entre el 98\% y el 102\% del precio de la última transacción del período anterior $\left(P_{t-1}\right)$ :

$$
P_{t}^{i} \sim U\left(0,98 P_{t-1}, 1,02 P_{t-1}\right)
$$

En nuestro mercado, los AIC deberian contribuir al aumento del volumen del mercado ya que introducen discrepancia en la valoración del activo

3. Agentes Técnicos (AT). Son analistas técnicos que intentan identificar tendencias a partir de los precios. Al igual que los analistas técnicos, los AT calculan con cada transacción la media de los precios de transacción de los últimos 20 periodos (media móvil larga) y la media de los últimos 3 periodos (media móvil corta). Los AT desean comprar (vender) si y sólo si la media móvil corta es superior (inferior) a la larga, es decir si identifican una tendencia alcista (bajista) en la serie precios de transacción. Estos agentes no introducen órdenes límite. La razón es que el agente desea asegurarse la transacción antes de que cambie la tendencia.

4. Agentes con Redes Neuronal Artificiales (ARNA). Estos agentes forman sus expectativas sobre el precio del activo con riesgo de acuerdo a un modelo no 
lineal: empleando Redes Neuronales Artificiales. ${ }^{5}$ Los agentes utilizan los 10 últimos rendimientos observados en la red neuronal y tras un proceso de aprendizaje obtienen una predicción para el próximo periodo. Si este rendimiento predicho es positivo, los agentes toman una posición de compra y viceversa si es negativo.

Aunque existe una gran tipología de estructuras que puede tener una Red Neuronal Artificial, la más común es la red alimentada hacia delante o feedforward (para una información más detallada consultar Kuan y White, 1994), en la cual la información fluye en un único sentido desde la capa de entrada a la capa de salida pasando por la capa oculta. Esta es la estructura utilizada en nuestro trabajo, exactamente los agentes realizan sus predicciones a través de una red neuronal artificial feedforward con una sola capa oculta. Dentro de la red las unidades están conectadas por pesos sinápticos, los cuales determinan cuantitativamente la influencia de una unidad sobre la otra. El conjunto de todos los pesos de interconexión entre la unidades i y j, $W_{i j}$ es conocido como la matriz de ponderaciones. Para encontrar los pesos óptimos de esta matriz $\left(\mathrm{W}^{*}\right)$ se utiliza un procedimiento de prueba y error, generalmente denominado como aprendizaje. Este procedimiento de aprendizaje permite hallar los parámetros que minimizan los errores de la red durante un conjunto de datos de entrenamiento.

Al principio de cada simulación, cada agente recibe una dotación idéntica consistente en 1000 unidades monetarias y 10 unidades del activo con riesgo. Cada 120 períodos de negociación los agentes reciben de nuevo la dotación, que se suma a la riqueza alcanzada. Dado que los AT y los ARNA necesitan disponer de datos pasados para empezar a operar, hasta que no se alcance un número mínimo de transacciones sólo están presentes en el mercado los AIC y los AF. En el momento en que todos los agentes se encuentran operando en el mercado se reinicializa la riqueza. Por ultimo debemos indicar que los agentes no pueden abstenerse de introducir órdenes. ${ }^{6}$ Además, los precios se mantienen de un periodo a otro y la prioridad de órdenes con igual precio es el momento de llegada.

\section{Resultados}

\footnotetext{
${ }^{5}$ La predicción de precios en activos financieros ha constituido uno de los campos donde las RNAs más se han empleado dentro de las finanzas (véase, por ejemplo, Stock y Watson, 1999, o Harvey et al., 1999). ${ }^{6}$ Nótese que la introducción de órdenes tiene coste cero y por tanto no es optimo abstenerse.
} 
A continuación se presentan los resultados de las simulaciones. En primer lugar, se han simulado cien sendas de precios fundamentales de acuerdo a (1) con $\mu$ variable con cada senda (distribuida uniformemente entre $+/-5 \%$ ), $\sigma=10 \%$, y $P_{f, 0}=100$. La razón por la que permitimos que $\mu$ varíe, es evitar que los resultados estén condicionados a una tendencia concreta en la serie de precios, de forma que nuestros resultados sean independientes de si el mercado es alcista o bajista. En segundo lugar, para cada senda de precios fundamentales se han realizado cuatro simulaciones diferentes por cada distribución de agentes considerada (ver Cuadro 2), consistentes en 1.000 periodos (cuatro años) con un total de 5.000 rondas de negociación. $^{7}$

\subsection{Dinámica de precios de transacción}

En el Cuadro 3, se muestra el promedio para todas las simulaciones y para cada mercado de los estadísticos descriptivos correspondientes a las series de rendimientos (calculado como la diferencia entre el logaritmo natural de los precios de cierre en dos periodos consecutivos). En concreto, para cada serie de rendimientos se ha calculado su media anualizada, su desviación típica anualizada, el coeficiente de asimetría, el coeficiente de curtosis, el primer percentil o valor en riesgo, y si se rechaza o no la hipótesis de normalidad utilizando el contraste Jarque-Bera.

El Cuadro 3 muestra que, al igual que se obtiene en otros mercados financieros con agentes computacionales, nuestro mercado artificial genera rendimientos de transacción leptocúrticos y no normales. Es decir, aunque globalmente los precios de transacción no se aparten excesivamente de la senda fundamental, tal y como se ha mencionado en la subsección anterior, las propiedades estadísticas de las series de rendimientos difieren notablemente de las de los rendimientos fundamentales y se acercan a la evidencia empírica encontrada en los mercados financieros reales.

Curiosamente, aunque la presencia de AT (Mercado 2) y ARNA (Mercado 3) disminuye la volatilidad de los rendimientos con respecto al Mercado 1 (tanto en términos de desviación típica como en términos de valor en riesgo), el exceso de curtosis aumenta, especialmente en el caso de los ARNA. En otras palabras, aumenta el tamaño relativo de las colas en la distribución de rendimientos. Cuando en el mercado operan tanto AT como ARNA, no sólo disminuye la volatilidad sino también el exceso

\footnotetext{
${ }^{7}$ Los resultados presentados corresponden a los periodos en los que todos los agentes están activos.
} 
de curtosis, posiblemente debido a la mayor heterogeneidad en cuanto a la composición de los agentes.

Para ilustrar lo anterior, en los Gráficos 1 a 3 se muestran las series de precios, las series de rendimientos, y la función de densidad estimada de los rendimientos estandarizados para los cuatro mercados y una senda fundamental concreta. ${ }^{8}$ En ellos se puede apreciar visualmente (para este ejemplo concreto) tanto el grado de eficiencia del mercado, como la distinta volatilidad de las series de rendimientos, así como la curtosis de los rendimientos de transacción frente a la normalidad de los rendimientos fundamentales.

Otra propiedad de las series de rendimientos reales es la presencia de cierto grado de correlación serial o predecibilidad en los mismos. El Cuadro 4 muestra coeficientes de correlación serial medios para cada mercado. En los cuatro mercados, los rendimientos exhiben correlación serial negativa significativa en el primer retardo, y se rechaza en la práctica totalidad de simulaciones la hipótesis nula de ausencia de correlación serial en los primeros 5 y 10 retardos mediante el contraste de Ljung-Box. Esto se debe posiblemente al hecho de que cuando el precio de transacción cae por debajo del valor fundamental tiende a regresar a la senda fundamental en el siguiente periodo debido a la demanda de los AF, luego el rendimiento negativo de este tipo en un periodo tiende a inducir un rendimiento de signo contrario en el siguiente periodo. Nuestros resultados coinciden hasta cierto punto con lo que sucede en los mercados reales. Campbell, Grossman y Wang (1993), por ejemplo, encuentran que los rendimientos están serialmente negativamente correlacionados, pero únicamente con posterioridad a períodos de gran volumen de negociación, que los autores relacionan con las ventas de agentes no informados.

Cuando los AT y los ARNA operan en el mercado, la correlación serial negativa se atenúa, debido posiblemente al aumento de la eficiencia de los precios.

Por último, hemos empleado el contraste de Engle (1982) para la hipótesis nula de ausencia de efectos ARCH en la serie de residuos resultantes de regresar los rendimientos sobre sus retardos significativos. El Cuadro 5 muestra los resultados. En el Mercado1 (sólo AIC y AF) se rechaza la hipótesis nula con mayor frecuencia que el nivel de significación, aunque la presencia de efectos ARCH es mucho más evidente según consideramos los Mercados 2, 3 y 4 sucesivamente. Este resultado implica que la

\footnotetext{
${ }^{8}$ Para estimar la función de densidad se ha empleado un kernel Nadaraya-Watson gaussiano.
} 
persistencia en la volatilidad condicionada es un resultado robusto al mecanismo de negociación empleado y al proceso de llegada de información al mercado, ya que también está ampliamente documentado en mercados artificiales distintos al nuestro (véase, por ejemplo, LeBaron et al., 1999). Sin embargo, el resultado más novedoso es que el fenómeno ARCH está vinculado a la presencia de agentes técnicos, y sobre todo, de agentes con capacidad de aprendizaje. En el mercado 4, en el que ambos tipos de agentes operan junto a los AF y AIC, el rechazo de la hipótesis es generalizado.

En resumen, la presencia de AT y ARNA no sólo aumenta la eficiencia informativa de los precios de transacción, sino que también disminuye la volatilidad, aumenta el exceso de curtosis, disminuye la correlación serial negativa, y aumenta los efectos ARCH.

\subsection{Eficiencia informativa}

Con el fin de medir el efecto de cambios en la población de agentes sobre la eficiencia informativa del mercado, empleamos tres medidas de eficiencia basadas en una comparación entre el valor fundamental del activo y el precio de la última transacción de cada periodo (Theissen, 2000). ${ }^{9}$

a) Error absoluto medio.

Es el promedio del valor absoluto de las desviaciones del precio de cierre con respecto al precio fundamental del periodo:

$$
E A M=\frac{1}{950} \sum_{t=51}^{1000}\left|P_{t}-P_{f, t}\right|
$$

b) Error relativo medio.

Permite comparar la eficiencia con independencia del nivel de precios:

$$
E R M=\frac{1}{950} \sum_{t=51}^{1000} \frac{\left|P_{t}-P_{f, t}\right|}{P_{f, t}}
$$

c) Raíz del error cuadrático medio.

Da mayor peso a las desviaciones mayores:

$$
R E C M=\sqrt{\frac{1}{950} \sum_{t=51}^{1000}\left(P_{t}-P_{f, t}\right)^{2}}
$$

\footnotetext{
${ }^{9}$ En todos los casos, se descartan las 50 primeras observaciones con el fin de considerar únicamente aquellos periodos en los que todos los agentes de cada mercado están operando.
} 
El Cuadro 6 presenta el promedio de las tres medidas de eficiencia para las 100 simulaciones para cada tipo de mercado. La primera conclusión que se puede extraer del análisis de eficiencia es que el mecanismo de negociación de doble subasta continua permite una diseminación razonable de la información privada para una fracción suficiente de agentes con información sobre el valor fundamental. El error relativo medio en el Mercado 1 es en promedio apenas un 1\%, a pesar de que el $80 \%$ de los agentes no observan-ni siquiera con ruido-el proceso fundamental. El elevado RECM (2.578) sugiere, sin embargo, que en este mercado se cierran transacciones a precios alejados de la senda fundamental. Ello es debido a la heterogeneidad en las creencias de los AIC, así como al intento de los AF de explotarlas en su beneficio.

Cuando 20 de los agentes con inteligencia cero son sustituidos por agentes técnicos (Mercado 2) o agentes con redes neuronales artificiales (Mercado 3), la eficiencia de los precios de transacción mejora ostensiblemente, muy especialmente en el Mercado 3. Este resultado es indicativo de que el análisis de precios pasados permite a los agentes generar predicciones mejores sobre el valor fundamental que las de los agentes con inteligencia cero. Lo interesante, sin embargo, es que la presencia tanto de AT como de ARNA, que intentan explotar regularidades en los precios (ineficiencias), tiene el efecto de facilitar la diseminación de la información fundamental, y por tanto de incrementar la eficiencia del mismo.

La mejora de eficiencia se aprecia especialmente en el caso de los agentes ARNA, que reducen el EAM y el ERM en un 40\%, y la RECM en más de un 60\% con respecto al Mercado 1. Por último, cuando las cuatro clases de agentes operan conjuntamente (Mercado 4), la eficiencia informativa de los precios de transacción aumenta un 60\% en términos de EAM y ERM, y más de un 80\% en términos de RECM.

Aunque las conclusiones deben ser interpretadas con cautela dado que solamente comparamos cuatro mercados con una determinada distribución de agentes, la eficiencia en nuestro mercado aumenta con la presencia de AT y ARNA a pesar de que el porcentaje de agentes fundamentales permanece constante. Este resultado es consistente con el hecho de que el mercado 4 contiene el menor numero de agentes con Inteligencia Cero, el mercado 3 no tiene agentes técnicos y el 2 no contiene a los ARNA. De modo que según pasamos de un mercado a otro la eficiencia aumenta como consecuencia del incremento relativo de agentes que predicen con menor error. 


\subsection{Volumen de negociación}

Además de la dinámica de precios, resulta interesante estudiar la liquidez de los distintos mercados y su evolución a lo largo del tiempo. En el Gráfico 4 se muestra el promedio para las 100 simulaciones del número de unidades del activo con riesgo intercambiadas en cada periodo de negociación. En los cuatro mercados se repite un patrón semejante. El mercado comienza con un número modesto de transacciones que, o bien se mantiene, o bien se reduce en los siguientes períodos, hasta que los agentes reciben una nueva dotación de unidades monetarias y unidades del activo, momento en el cual el volumen de negociación salta a un nivel superior. ${ }^{10}$ En los siguientes periodos se repite este patrón hasta que en todos los mercados el volumen de negociación se estabiliza y ya no es sensible al aumento de renta y unidades del activo en manos del conjunto de agentes alcanzándose un “equilibrio”. Este comportamiento sugiere que la restricción presupuestaria es efectiva para los bajos niveles de riqueza de los primeros periodos y apenas afecta al volumen de liquidez para niveles superiores de riqueza.

En cuanto al efecto de cambios en la población de agentes sobre el volumen del mercado, la sustitución de AIC por AT (Mercado 2) y ARNA (Mercado 3) tiene como consecuencia una disminución en la liquidez del mercado, tal y como es de esperar, dado que el papel de los AIC es precisamente el de dotar de heterogeneidad suficiente en las creencias de los agentes y aumentar por tanto el número de transacciones. Lo interesante, sin embargo, es que esa reducción en liquidez es mucho mayor en el caso de los AT. Ello es posiblemente debido a que los AT no envían órdenes límite.

\subsection{Riqueza de los agentes}

Es interesante analizar la distribución de la riqueza (y composición de las cartera) que se produce en un mercado con información asimétrica (o incluso donde existe un pequeño grupo de agentes con información privilegiada) y cómo evoluciona según entran en el mercado nuevos agentes no informados pero con capacidad de explotar la información contenida en los precios de transacción pasados.

Para estudiar la evolución de la riqueza, se ha calculado en cada periodo de negociación de cada simulación la media de las unidades monetarias, unidades del activo y riqueza total de cada clase de agente. Posteriormente, se ha calculado el

\footnotetext{
${ }^{10}$ Los resultados se documentan en todos los casos a partir del periodo de negociación en que todos los agentes correspondientes a ese mercado están operando.
} 
promedio de las cien simulaciones, que se recogen en los Gráficos 5 a 8, correspondientes a los mercados 1 a 4 .

El Gráfico 5 muestra los resultados para el mercado en el que operan 80 AIC y 20 AF (Mercado 1). Como se ha visto, los AF son capaces de conducir el precio de transacción al precio fundamental, que sólo ellos observan (con un pequeño ruido). Por tanto, no es poco frecuente que compren por debajo del precio fundamental a los AIC con predicciones más bajas y vendan tras el correspondiente ajuste a un precio superior. En consecuencia, los AF tienen la capacidad de acumular unidades del activo con riesgo y financiarlas con las ganancias derivadas de su interacción con los AIC, de forma que su efectivo también aumenta.

Pero más interesante es analizar qué ocurre cuando agentes no informados pero que explotan la información pasada disponible entran en el mercado, es decir, cuando agentes que utilizan análisis técnico o modelos estadísticos entran a negociar. En el Gráfico 6 puede observarse lo que ocurre cuando los AT sustituyen a 20 AIC. Los AT tienden a mantener en promedio la misma posición en el activo que los AIC, sin embargo, su posición en efectivo es notablemente superior. Este resultado sugiere que los AT, mediante el análisis de la evolución de los precios tienen una capacidad considerable de detectar cuándo el activo esta infravalorado o sobrevalorado, lo cual les permite obtener ganancias en sus compras y ventas con los AIC. Es interesante destacar que éstos no están peor que en el Mercado 1, mientras que los AF pierden parte de su ventaja en efectivo a favor de los AT.

El Gráfico 7 corresponde al Mercado 3, en el que operan 60 AIC, 20 AF y 20 ARNA. La evolución de éstos últimos no difiere demasiado de la de los AT en el Mercado 2: mantienen posiciones en el activo con riesgo semejantes a los AIC, pero posiciones en efectivo mucho mayores. En este caso, el detrimento en la posición en efectivo que sufren los AF es mayor que en el caso anterior. La mayor riqueza alcanzada por los ARNA, confirma la superioridad de su regla de predicción con respecto a la que utilizan los AT, lo cual es consistente con la mayor eficiencia del Mercado 3 con respecto al Mercado 2.

Por último, cuando las cuatro clases de agentes están presentes (Mercado 4), el Gráfico 8 muestra que los AF no sólo disponen de menos efectivo que en el Mercado 1, sino que se ven obligados a disminuir de manera apreciable su posición en el activo con riesgo. Como consecuencia, la ventaja en riqueza de los AF con respecto al resto de agentes disminuye notablemente. En el Gráfico, también se aprecia la superioridad de 
los ARNA con respecto a los AT, puesto que ambos grupos mantienen en promedio posiciones similares en el activo con riesgo, pero los primeros disponen sistemáticamente de mayores posiciones en el activo libre de riesgo.

En resumen, los AF tienen la capacidad de transmitir al mercado su información privada y beneficiarse de la eficiencia de los precios de transacción. Al hacerlo, sin embargo, están creando una externalidad positiva que potencialmente pueden utilizar algunos agentes como los AT y muy especialmente los ARNA, lo cual disminuye el valor que les reporta su información privada.En un mercado en el que los precios fuesen siempre distintos al fundamental los AF no podrían deshacer sus posiciones y obtener un beneficio. Por tanto es cierto que la ineficiencia contribuye pero necesitamos que en algún momento los precios de transacción vuelvan a ser eficientes.

\section{Resumen y conclusiones}

En este trabajo, hemos presentado un nuevo modelo de mercado de agentes computacionales. En nuestro mercado, cuyo mecanismo de negociación es una doble subasta continua, no sólo existe información asimétrica ex-ante, ya que la mayoría de agentes no disponen de información alguna sobre el valor fundamental del activo, sino también ex-post, de forma que la única fuente de información sobre el valor fundamental para los agentes no informados son los propios precios de transacción pasados. Además, a diferencia de otros trabajos, permitimos que el valor fundamental del activo evolucione en el tiempo de acuerdo a un proceso estocástico, lo cual permite calibrar el modelo a datos reales. Por último, analizamos el efecto de que agentes no informados pero que utilizan la información pasada entren este mercado.

Los resultados de 100 simulaciones de 1.000 periodos cada una en cuatro mercados distintos, permiten extraer una serie de conclusiones. La primera es que el mecanismo de doble subasta continua permite potencialmente una correcta diseminación de la información privada con respecto al proceso fundamental. En segundo lugar, los rendimientos generados por este mercado reproducen los hechos estilizados que exhiben las distribuciones de rendimientos reales: exceso de curtosis, no normalidad, predecibilidad, y efectos ARCH. En tercer lugar, la presencia de analistas técnicos y agentes que aprenden de precios pasados mediante el uso de redes neuronales artificiales, mejora notablemente la eficiencia informativa del mercado. 
En concreto, los agentes técnicos, y sobre todo los agentes con redes neuronales artificiales, contribuyen a disminuir la volatilidad del mercado y el grado de correlación serial negativa, pero aumentan la persistencia de la heteroscedasticidad condicionada.

Por último, el análisis de la riqueza de los agentes demuestra que el análisis técnico en este mercado, y sobre todo el uso de redes neuronales artificiales, permite obtener mejores predicciones que las de los agentes con inteligencia cero y extraer parasitariamente parte de las rentas de los agentes fundamentales, que son quienes proveen al mercado de información relativa al verdadero valor fundamental del activo.

En cuanto a investigación futura, pensamos que nuestro mercado artificial constituye la base de una potente herramienta que nos permitirá estudiar un amplio número de problemas de microestructura en un mercado artificial que intente captar la complejidad de los mercados reales. En cuanto al propio diseño del mercado podemos destacar algunas extensiones posibles. En primer lugar, el hecho de que nuestros agentes unicamente pueden realizar transacciones de una unidad del activo con riesgo cada vez, impide estudiar el impacto del tamaño de la transacción sobre el precio. Obviamente, en este caso, sería necesario relajar el supuesto de neutralidad al riesgo para permitir que exista una solución interior al problema de optimización de cartera del inversor. En segundo lugar, la restricción puede jugar un papel fundamental tanto sobre la eficiencia como sobre la propia dinámica de precios. Por ejemplo, la capacidad de influir en los precios de los agentes informados está limitada por su restricción presupuestaria. Por ultimo, la diversidad de arquitecturas en la red neuronal permitiría enriquecer el análisis. 


\section{Referencias Bibliográficas}

Ackert, Lucy F., Bryan Church y Narayanan Jayaraman. (2001) "An Experimental Study Of Circuit Breakers: The Effects Of Mandated Market Closures And Temporary Halts On Market Behavior," Journal of Financial Markets, 4, 185201.

Arthur, W.B., Holland, J., LeBaron, B., Palmer, R., Tayler, P. (1997) “Asset Pricing Under Endogenous Expectations in An Artifficial Stock Market.” En: Arthur, W.B., Durlauf, S., Lane, D. (editores), The Economy as an Evolving Complex System II. Addison-Wesley, Reading, MA, 15-44.

Bray, M. (1982) "Learning, Estimation, and the Stability of Rational Expectations," Journal of Economic Theory, 26, 318-339.

Campbell, J., S. Grossman, y J. Wang. (1993) “Trading Volumen and Serial Correlation in Stock Returns,” Quarterly Journal of Economics, 108, 905-939.

Chan, Nicholas T., Blake D. LeBaron, Tomaso Poggio, y Andrew W. Lo. (2001) "Agent-Based Models of Financial Markets: A Comparison with Experimental Markets,” MIT Sloan Working Paper No. 4195-01.

Chiarella, C. y G. Iori. (2002) "A Simulation Analysis of the Microstructure of Double Auction Markets,” Quantitative Finance, 2, 346-353.

Engle, R. F. (1982) "Autoregressive Conditional Heteroskedasticity with Estimates of the Variance of United Kingdom Inflation," Econometrica, 50, 987-1007.

Gode, D. K. y S. Sunder. (1993) "Allocative Efficiency of Markets with Zero Intelligence Traders,” Journal of Political Economy 101, 119-137.

Grossman, S. J., y J. E. Stiglitz. (1980) “On the Impossibility of Informationally Efficient Markets,” American Economic Review, 70, 393-408.

Harvey, C. R., K.E Travers,. y J.M. Costa. (1999) "Forecasting Emerging Market Returns Using Neural Networks”, Financial Technology, 25-35.

Hornik, K., M. Stinchcombe y H. White, 1989, “Multilayer feedforward networks are universal approximators”, Neural Networks, 2, 359-366.

Iori, G. (2002) “A microsimulation of Traders Activity in the Stock Market: The Role of Heterogeneity, Agents' Interactions and Trade Frictions," Journal of Economic Behaviour and Organization, 49, 271-287.

Kuan, C.M. y H. White (1994) “Artificial Neural Networks: an Econometric Perspective,” Econometric Reviews, 13, 1-91.

Kyle, A. (1985) "Continuous Auctions and Insider Trading,” Econometrica 53, 13151335.

LeBaron, B., W. B. Arthur, y R. Palmer (1999) “Time Series Properties of an Artificial Stock Market,” Journal of Economic Dynamics and Control 23, 1487-1516.

LeBaron, B. (2000) “Agent-Based Computational Finance: Suggested Readings and Early Research,” Journal of Economic Dynamics and Control, 24, 679-702.

LeBaron, B. (2001) “A Builder's Guide to Agent-Based Financial Markets,” Quantitative Finance, 1, 254-261. 
LeBaron, B. (2002) "Building the Santa Fe Artificial Stock Market,” Working Paper, Brandeis University.

Madhavan, A. (2000) “Market Microstructure: A Survey,” Journal of Financial Markets 3, 205-258.

Robbins, H. y S. Monro, (1951) “A Stochastic Approximation Method”, The Annals of Mathematical Statistics, 22, 400-407.

Stock, James H. y M. W. Watson. (1999) "A Comparison of Linear and Nonlinear Univariate Models for Forecasting Macroeconomic Time Series," ch. 1 in R. Engle and H. White (eds.), Cointegration, Causality and Forecasting: A Festschrift for Clive W.J. Granger. Oxford: Oxford University Press.

Theissen, E. (2000) "Market Structure, Informational Efficiency and Liquidity: An Experimental Comparison of Auction and Dealer Markets,” Journal of Financial Markets, 3, 333-363.

Yang, J. (2002) “The Efficiency of an Artificial Double Auction Stock Market with Neural Learning Agents.” En Evolutionary Computation in Economics and Finance, editado por S.H. Chen, 79-98. Springer-Verlag. 


\section{Cuadro 1. Procedimiento de generación de órdenes.}

\begin{tabular}{|c|c|}
\hline Escenario & Acción \\
\hline \multicolumn{2}{|c|}{ Caso 1: existen precios de oferta (a) y demanda(b) } \\
\hline$P_{t}^{i}>a$ & Orden de mercado de compra \\
\hline$P_{t}^{i}<b$ & Orden de mercado de venta \\
\hline$b<P_{t}^{i}<a$ y $a-P_{t}^{i}>P_{t}^{i}-b$ & $\begin{array}{l}\text { Introduce una orden límite de venta } \\
{\left[\mathrm{U}\left(P_{t}^{i}, P_{t}^{i}+\mathrm{S}\right)\right]}\end{array}$ \\
\hline$b<P_{t}^{i}<a \quad$ y $\quad a-P_{t}^{i} \leq P_{t}^{i}-b$ & $\begin{array}{l}\text { Introduce una orden límite de compra } \\
{\left[\mathrm{U}\left(P_{t}^{i}-\mathrm{S}, P_{t}^{i}\right)\right]}\end{array}$ \\
\hline \multicolumn{2}{|l|}{ Caso 2: existe sólo precio de oferta (a) } \\
\hline$P_{t}^{i}>a$ & Orden de mercado de compra \\
\hline$P_{t}^{i} \leq a$ & Orden límite de compra \\
\hline \multicolumn{2}{|l|}{ Caso 3: existe sólo precio de demanda (b) } \\
\hline$P_{t}^{i}<b$ & Orden de mercado de venta \\
\hline$P_{t}^{i} \geq b$ & Orden limite de venta \\
\hline \multicolumn{2}{|l|}{ Caso 4: no existe precio de oferta ni de demanda } \\
\hline Con probabilidad $1 / 2$ & Orden límite de compra \\
\hline Con probabilidad $1 / 2$ & Orden limite de venta \\
\hline
\end{tabular}

Cuadro 2. Distribución de agentes en cada mercado.

\begin{tabular}{lcccc}
\hline & $\begin{array}{c}\text { Agentes con } \\
\text { Inteligencia Cero }\end{array}$ & $\begin{array}{c}\text { Agentes } \\
\text { Fundamentales }\end{array}$ & $\begin{array}{c}\text { Agentes } \\
\text { Técnicos }\end{array}$ & $\begin{array}{c}\text { Agentes con Redes } \\
\text { Neuronales Artificiales }\end{array}$ \\
\hline Mercado 1 & 80 & 20 & 0 & 0 \\
Mercado 2 & 60 & 20 & 20 & 0 \\
Mercado 3 & 60 & 20 & 0 & 20 \\
Mercado 4 & 40 & 20 & 20 & 20 \\
\hline
\end{tabular}




\section{Cuadro 3. Estadísticos descriptivos de los rendimientos.}

El Cuadro muestra en la columna 2 el promedio para las cien simulaciones del rendimiento medio entre precios de cierre multiplicado por 250; en la columna 3 el promedio de la desviación típica de los rendimientos multiplicada por la raíz de 250; en la columna 4, el promedio del coeficiente de asimetría; en la columna 5, el promedio del coeficiente de curtosis; en la columna 6, el promedio del valor en riesgo (Value-at-Risk) en tanto por ciento, calculado como el percentil más bajo de la serie de rendimientos; y en las columna 7 y 8, el porcentaje de simulaciones para las cuales el contraste de Jarque-Bera rechaza la hipótesis nula de normalidad a los niveles de significación del 5\% y el 1\% respectivamente. La fila 2 corresponde al proceso de rendimientos fundamental, mientras que las filas 3 a 6 muestran los resultados para los rendimientos de transacción simulados en distintos mercados.

\begin{tabular}{cccccccc}
\hline & $\begin{array}{c}\text { Rendimiento } \\
\text { Medio } \\
\text { Anualizado }\end{array}$ & $\begin{array}{c}\text { Desviación } \\
\text { Típica } \\
\text { Anualizada }\end{array}$ & $\begin{array}{c}\text { Coeficiente de } \\
\text { Asimetría }\end{array}$ & $\begin{array}{c}\text { Coeficiente de } \\
\text { Curtosis }\end{array}$ & VaR & $\begin{array}{c}\text { Rechazo } \\
\text { Normalidad al } \\
5 \%\end{array}$ & $\begin{array}{c}\text { Rechazo } \\
\text { Normalidad al 1\% }\end{array}$ \\
\hline Fundamental & -0.0083 & 0.0997 & 0.0168 & 2.9756 & 2.0200 & 6 & 0 \\
Mercado 1 & -0.0079 & 0.3700 & -0.0009 & 4.4904 & 9.8200 & 99 & 99 \\
Mercado 2 & -0.0078 & 0.2957 & 0.0187 & 4.8111 & 8.4400 & 99 & 99 \\
Mercado 3 & -0.0078 & 0.2342 & 0.0078 & 4.9156 & 6.6800 & 100 & 99 \\
Mercado 4 & -0.0078 & 0.1742 & -0.0177 & 4.7034 & 4.9100 & 99 & 98
\end{tabular}




\section{Cuadro 4. Correlación serial de rendimientos.}

El Cuadro muestra el promedio para las 100 simulaciones por cada mercado de los coeficientes de correlación serial de orden $\tau\left(\rho_{\tau}\right)$, así como el porcentaje de rechazos de la hipótesis nula de ausencia de correlación serial en los 5 y 10 primeros retardos al 5\% de significación.

\begin{tabular}{ccccccc}
\hline & $\rho_{1}$ & $\rho_{2}$ & $\rho_{3}$ & $\rho_{4}$ & $\begin{array}{c}\text { Rechazo 5 } \\
\text { retardos al 5\% }\end{array}$ & $\begin{array}{c}\text { Rechazo 10 } \\
\text { retardos al 5\% }\end{array}$ \\
\hline Fundamental & 0.0012 & -0.0032 & 0.0049 & 0.0005 & 6 & 7 \\
Mercado 1 & -0.4623 & 0.0001 & -0.0047 & 0.0038 & 100 & 100 \\
Mercado 2 & -0.4421 & -0.0032 & 0.0102 & -0.0009 & 100 & 100 \\
Mercado 3 & -0.4106 & 0.0006 & 0.0034 & -0.0017 & 100 & 100 \\
Mercado 4 & -0.3368 & 0.004 & 0.0025 & -0.0027 & 100 & 100 \\
\hline
\end{tabular}

\section{Cuadro 5. Efectos ARCH.}

\begin{tabular}{ccc}
\hline & $\begin{array}{c}\text { Rechazo } \\
\text { Homosc. } \\
(5 \%)\end{array}$ & $\begin{array}{c}\text { Rechazo } \\
\text { Homosc. } \\
(1 \%)\end{array}$ \\
\hline Fundamental & 9 & 2 \\
Mercado 1 & 34 & 22 \\
Mercado 2 & 56 & 37 \\
Mercado 3 & 76 & 52 \\
Mercado 4 & 93 & 88 \\
\hline
\end{tabular}

Cuadro 6. Eficiencia Informativa. El Cuadro muestra los promedios de las tres medidas de eficiencia para cada mercado.

\begin{tabular}{lccc}
\hline & Error Absoluto Medio & Error Relativo Medio & $\begin{array}{c}\text { Raíz del Error } \\
\text { Cuadrático Medio }\end{array}$ \\
\hline Mercado 1 & 1.0667 & 0.0107 & 2.578 \\
Mercado 2 & 0.8241 & 0.0083 & 1.5900 \\
Mercado 3 & 0.6349 & 0.0064 & 0.9374 \\
Mercado 4 & 0.4259 & 0.0043 & 0.4425 \\
\hline
\end{tabular}


Gráfico 1. Series de precios fundamentales y precios de transacción en los cuatro mercados para una senda fundamental concreta.
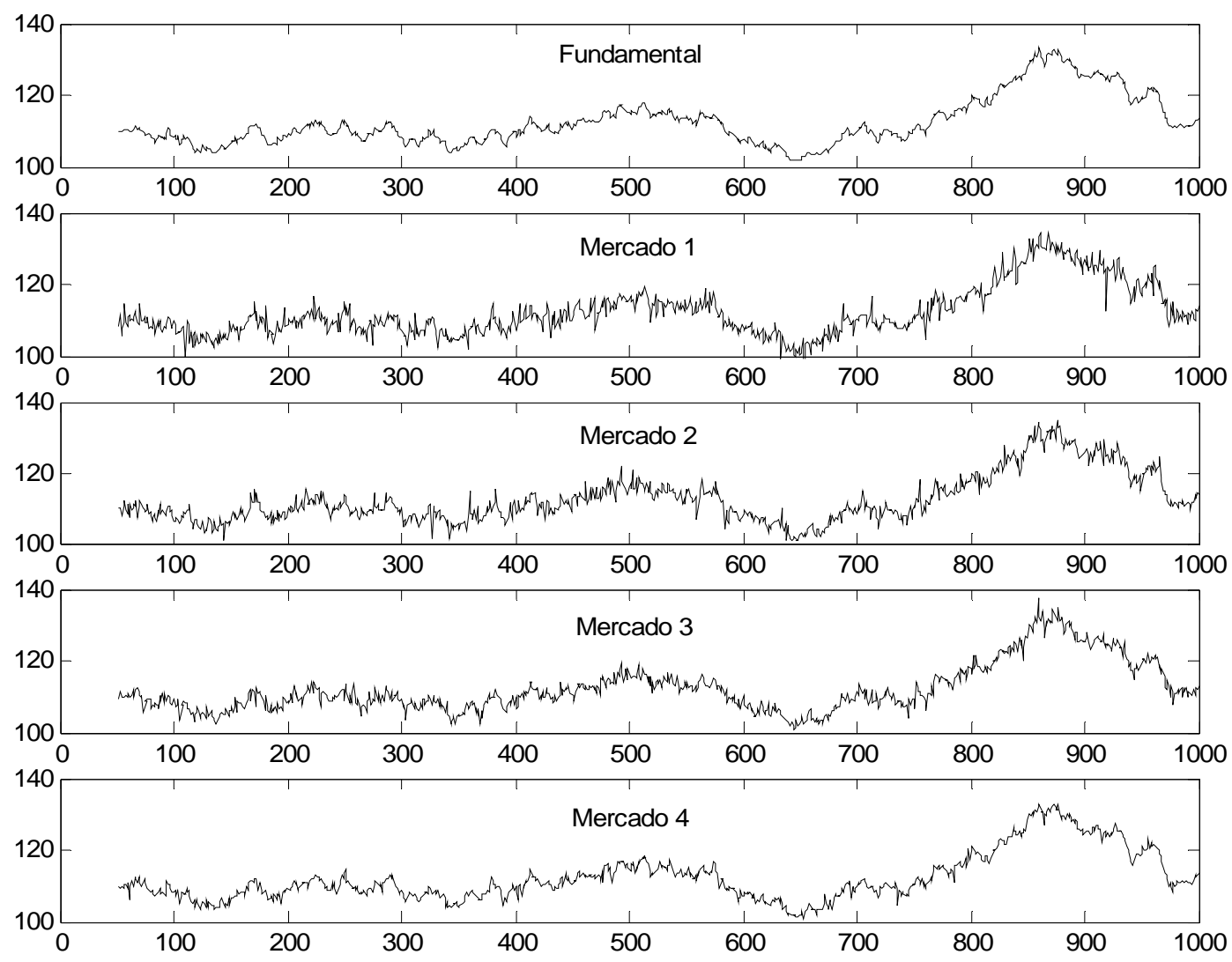
Gráfico 2. Series de rendimientos fundamentales y rendimientos de transacción en los cuatro mercados para una senda fundamental concreta.

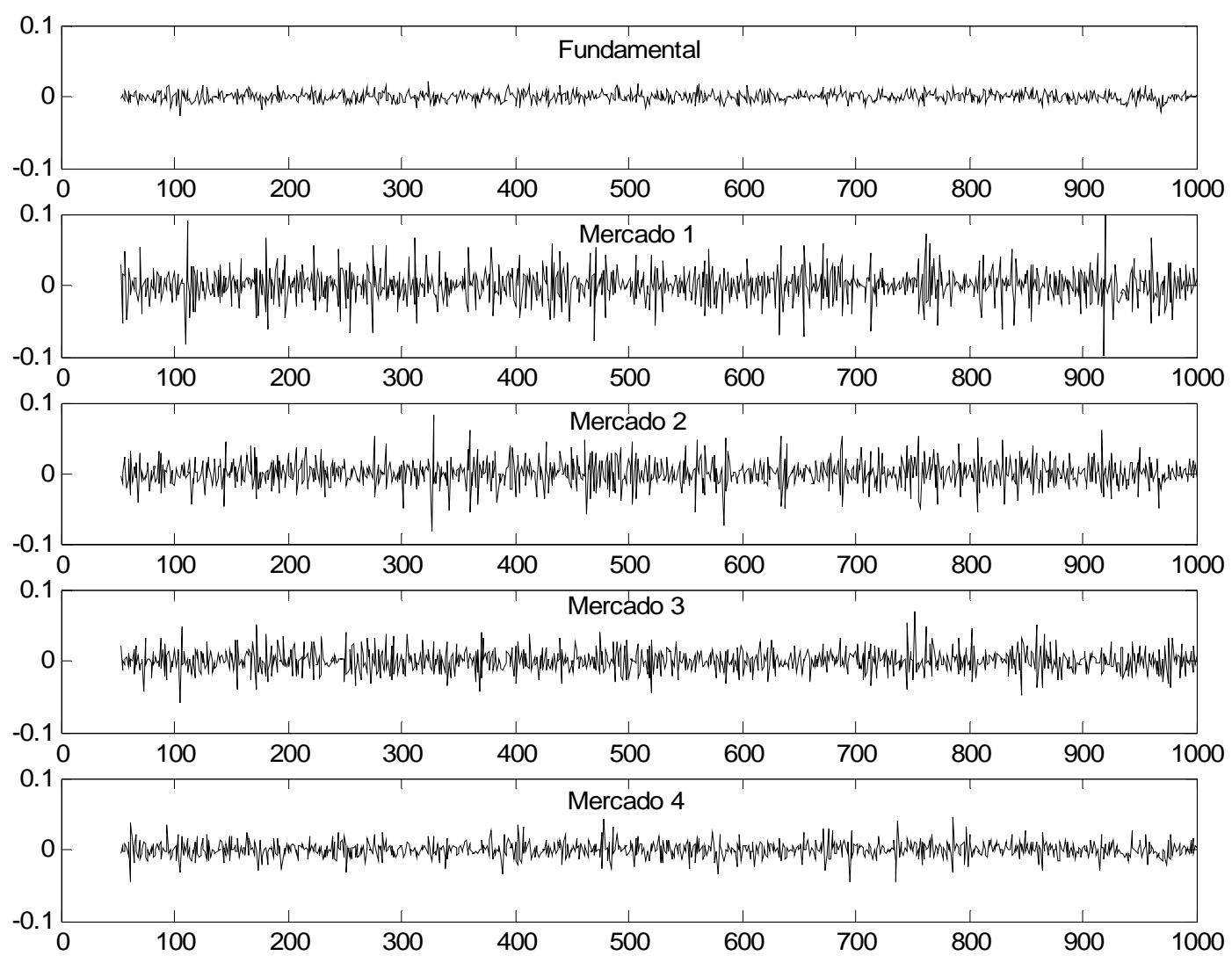


Gráfico 3. Funciones de densidad estimadas para las series de rendimientos fundamentales y rendimientos de transacción estandarizados en una senda fundamental concreta.

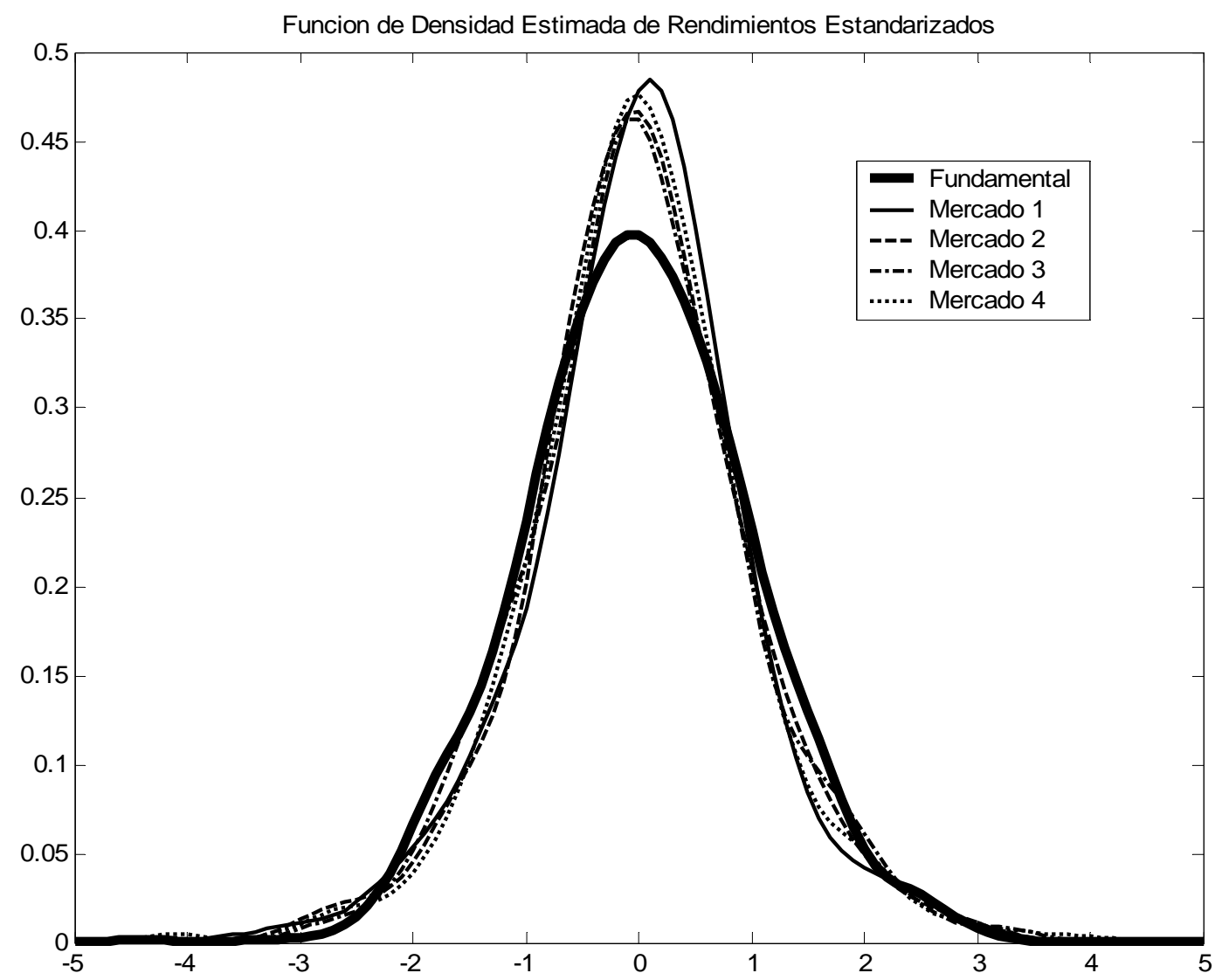




\section{Gráfico 4. Número de transacciones por periodo.}

El Gráfico muestra el promedio para las 100 simulaciones del número de unidades del activo con riesgo intercambiadas en cada periodo de negociación y en los cuatro mercados.

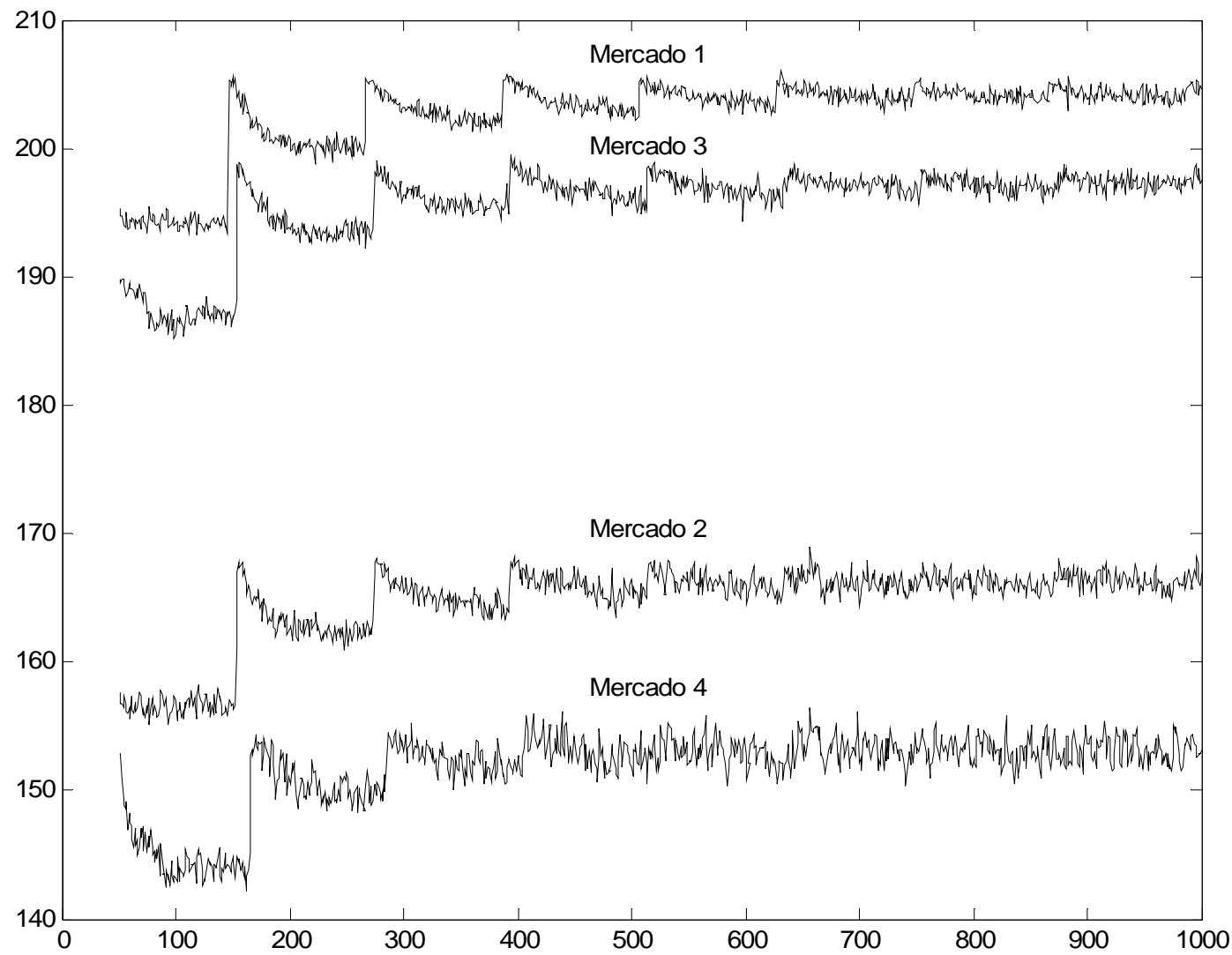


Gráfico 5. Evolución media de la riqueza de cada clase de agentes en el Mercado 1.

La línea gruesa representa la evolución a lo largo del tiempo de la riqueza de los Agentes con Inteligencia Cero y la fina corresponde a los Agentes Fundamentales.
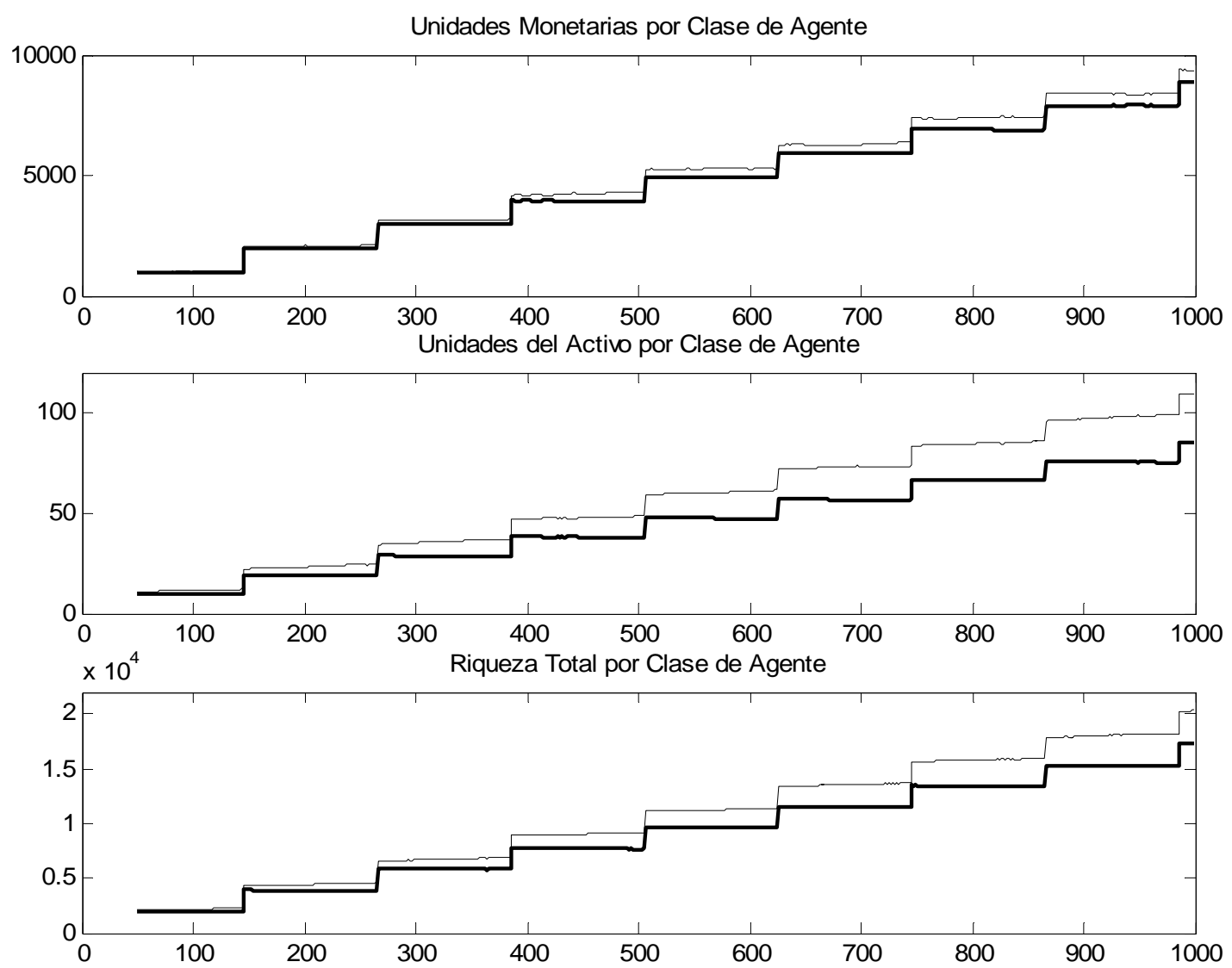
Gráfico 6. Evolución media de la riqueza de cada clase de agentes en el Mercado 2.

La línea gruesa representa la evolución a lo largo del tiempo de la riqueza de los Agentes con Inteligencia Cero, la fina, corresponde a los Agentes Fundamentales, y la línea a puntos a los Agentes Técnicos.
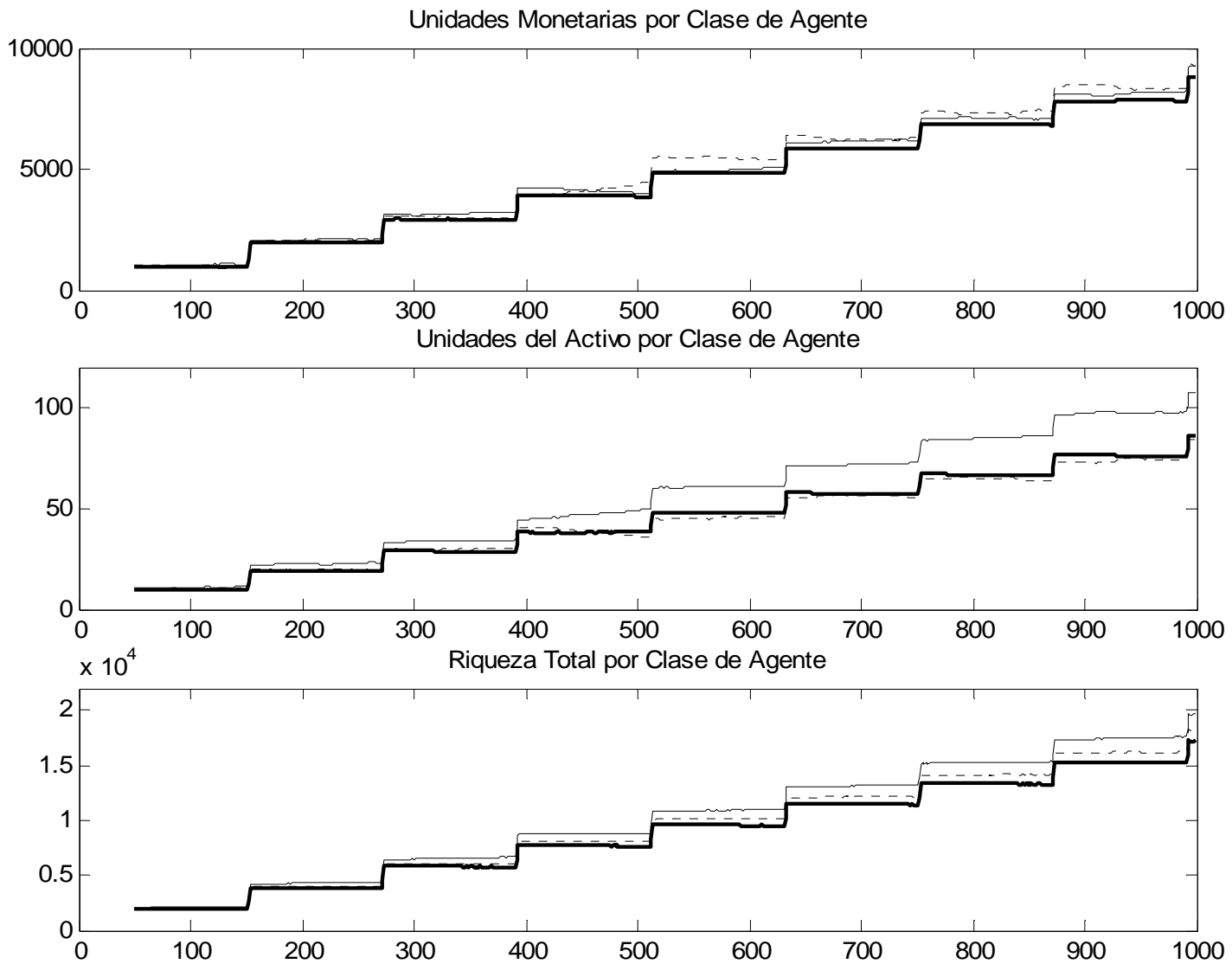
Gráfico 7. Evolución media de la riqueza de cada clase de agentes en el Mercado 3.

La línea gruesa representa la evolución a lo largo del tiempo de la riqueza de los Agentes con Inteligencia Cero, la fina, corresponde a los Agentes Fundamentales, y la línea a puntos a los Agentes con Redes Neuronales Artificiales.
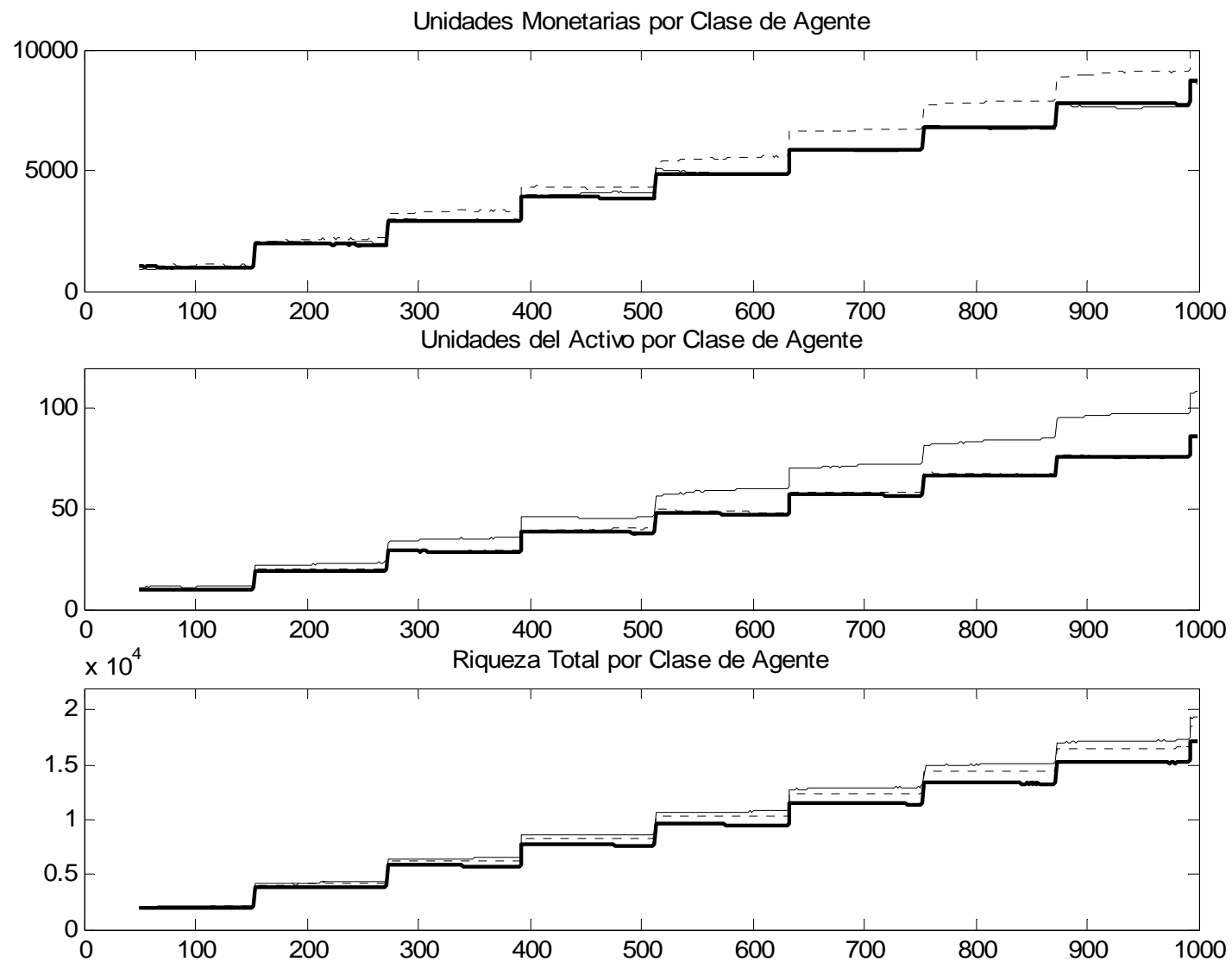
Gráfico 8. Evolución media de la riqueza de cada clase de agentes en el Mercado 4.

La línea gruesa representa la evolución a lo largo del tiempo de la riqueza de los Agentes con Inteligencia Cero, la fina, corresponde a los Agentes Fundamentales, la línea a rayas a los Agentes Técnicos y la línea a puntos a los Agentes con Redes Neuronales Artificiales.
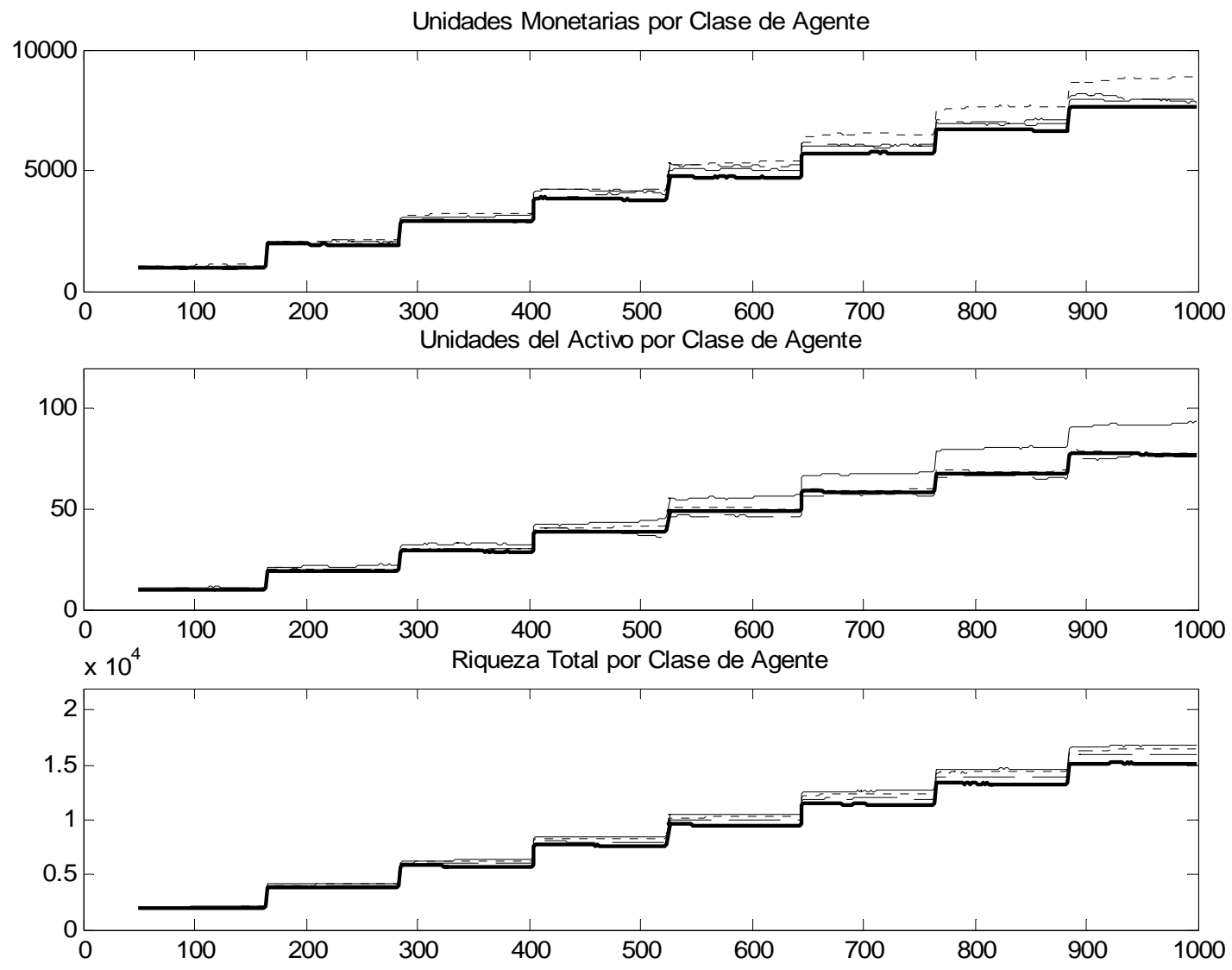